\title{
Micro mechanics of critical states for isotropically overconsolidated sand
}

\author{
G.R. McDowell, P. Yue, J.P. de Bono*
}

University of Nottingham, UK

\section{Abstract}

The discrete element method has been used to investigate the micro mechanics of shearing to a critical state on the loose and dense sides of critical. Isotropic compression has previously been modelled in 3D using a large number of particles and without the use of agglomerates. The same procedure is used here. Particle fracture is governed by the octahedral shear stress within the particle due to the multiple contacts and a Weibull distribution of strengths. Isotropic compression of a silica sand has been simulated to $20 \mathrm{MPa}$ and followed by unloading to a range of stresses before shearing to a critical state, using micro parameters which relate to the silica sand particle strengths. The samples at the lowest stress levels exhibit peak strength and dilation. The sample at the highest stress exhibits contraction and ductile yielding to a critical state. A critical state line is established, which appears to become parallel to the isotropic line in $\log e-\log p$ space at high stress levels. This paper shows that it is the evolving fractal particle size distribution during isotropic normal compression which governs the behaviour on unloading to different overconsolidation ratios. The micro mechanics of the critical state line are shown to be in the evolving particle size distribution during normal compression, and how such an aggregate behaves when it is unloaded.

\section{Introduction}

It has recently been demonstrated by the authors that it is possible to accurately model the onedimensional and isotropic compression of sand using the discrete element method [1,2]. It was shown that the normal compression line (NCL) for a sand should be linear when plotted on double- 
logarithmic axes, which confirmed earlier experimental and analytical propositions $[3,4]$. The compression index-that is, the slope of the compression line when plotted in log $e$-log $\sigma$ spacewas shown to be a function solely of the size-hardening law for the particles. For one-dimensional compression, the McDowell and de Bono [1] compression law is given by the equation:

$$
\log e=\log e_{\mathrm{y}}-\frac{1}{2 b} \log \frac{\sigma}{\sigma_{\mathrm{y}}}
$$

where $e$ is the current voids ratio, $\sigma$ is the applied stress, $e_{y}$ is the value on the linear log-log plot at a stress corresponding to the yield stress $\sigma_{y}$, and $b$ controls the size effect on average particle strength $\sigma_{\mathrm{av}}:$

$$
\sigma_{\mathrm{av}} \propto d^{-b}
$$

The normal compression lines for one-dimensional and isotropic compression were parallel, and the isotropic boundary conditions were shown to give rise to anisotropic local shear stresses within the soil sample.

De Bono and McDowell had previously implemented their simple breakage model to a large-scale triaxial model [5], with the aim of determining the nature and position of the critical state line for a crushable silica sand. Although their work demonstrated the effects and importance of crushing on critical states, only simulations with confining pressures up to $4 \mathrm{MPa}$ were shown, and they did not consider overconsolidated samples - in which fractal particle size distributions have developed as a result of normal compression. More recently, the authors have investigated the influence of particle shape and coordination number on the normal compression of granular soil, as well as highlighting some of the micro mechanics behind the emergence of fractal particle size distributions [6]. This 
short note is intended as a further step towards revealing the micro mechanics of the critical state using DEM.

By considering both a larger range of confining pressures, as well as overconsolidated sand, one of the key aims in this brief paper is to take the next step towards achieving a realistic critical state line in DEM, using a crushable sand with realistic characteristics -i.e. experimentally obtained strengths and hardening law, and the ability for particles to fragment repeatedly (a feat not possible using agglomerates). Another objective is to clarify whether the critical state line obtained from simple shear tests on the overconsolidated sand is parallel with the normal compression line defined by Equation (1). In the work presented here, a sample is compressed isotropically to $20 \mathrm{MPa}$, then unloaded to various stresses, resulting in samples with a range of overconsolidation ratios. These samples are then sheared (via conventional and constant- $p^{\prime}$ triaxial simulations) to critical states to reveal the critical state line in $\log e-\log p^{\prime}$ space (using a smaller, simpler triaxial model than in [5]). In addition, the lateral earth pressure coefficient, $K_{0}$, obtained from one-dimensional compression is used to predict a critical state angle of friction for the soil, which is then used to predict the critical state friction constant $M$ and compared to the data from the simulations.

\section{Modelling Particle Breakage}

The importance of including particle crushing when modelling granular soil is becoming increasingly recognised. Using DEM, crushing is typically modelled by either replacing breaking grains with new, smaller fragments [e.g. 7,8], sometimes referred to as the 'replacement method', or by using agglomerates [e.g. 9,10].

The crushing model used in this work uses the replacement method, and is detailed in McDowell and de Bono [1]. For brevity, only key details of the crushing model used here will be repeated; for full documentation of the model, as well as critical discussion of the choice of fracture criterion and breakage mechanism, readers are referred to prior publications $[1,2]$. 
Cheng et al. [11] presented similar simulations but using agglomerates (they isotropically compressed a sample to high stresses, then unloaded and sheared to states of constant volume). However, the work presented here is different in that by using the replacement method, the correct size effect on strength can be employed, and the particles can fragment indefinitely (there is no artificial comminution limit). A further drawback of using agglomerates is that the internal voids of the agglomerates rapidly become external voids once breakage commences. This means that the quantitative measure of voids ratio is meaningless and that the correct slope of the normal compression line cannot be obtained.

It is also important to note that without particle crushing, it is not possible to achieve a true normal compression line; Minh and Cheng [12] showed that for soils with a variety of gradings, a normal compression-like curve could be obtained using elastic unbreakable spheres; however, unloading such a sample, e.g. Bolton et al. [13] would result in voids ratio similar to, if not the same as the starting voids ratio, and so is not a normal compression line because the deformation is recoverable.

To determine whether fracture of a particle should occur or not, the average octahedral shear stress within each particle is used:

$$
q=\frac{1}{3}\left[\left(\sigma_{1}-\sigma_{2}\right)^{2}+\left(\sigma_{2}-\sigma_{3}\right)^{2}+\left(\sigma_{1}-\sigma_{3}\right)^{2}\right]^{1 / 2}
$$

which is calculated from the average principal stresses $\left(\sigma_{1}, \sigma_{2}, \sigma_{3}\right)$ in each particle. This method provides a simple criterion to facilitate breakage taking into account multiple contacts as well as different contact forces on a particle surface, while avoiding the use of computationally-demanding agglomerates-which are porous, cannot give quantitatively the correct normal compression line, require large numbers of balls to model the size effect on strength, and which give a comminution limit at relatively low stresses. 
McDowell [14] measured the tensile strength of sand grains by diametral compression between flat platens as $\sigma=F / d^{2}[15]$, and showed that the stresses at failure for a given particle size satisfied a Weibull distribution of strengths. These distributions were described by a characteristic value of strength, $\sigma_{0}$ and a Weibull modulus, $m$. In PFC3D [16], the software used here, the value of octahedral shear stress, $q$ induced in a spherical particle compressed diametrically between two walls is proportional to $F / d^{2}[2]$; hence, it is assumed that for a particle under multiple contacts, the particle will break if the octahedral shear stress is greater than or equal to its strength, where the strengths of the particles satisfy a Weibull distribution of $q$ values. Assuming the Weibull size effect is applicable to soil particles [e.g. 17], then the size-hardening parameter $b$ in Equation (2) is equal to $(3 / \mathrm{m})$. Rewriting this equation in terms of octahedral shear strength the particle strengths can be related to size by:

$$
q_{0} \propto d^{-\frac{3}{m}}
$$

where $q_{0}$ is the characteristic particle strength, and is a value of the distribution such that $37 \%$ (exp[-1]) of random strengths are greater (and for a given $m$ is proportional to the mean); and $m$ is the modulus (which is related to the coefficient of variation). It is this equation that is used when attributing random strengths to new fragments.

When a particle breaks, it is replaced by two new fragments, both of which are spheres. The new sphere fragments overlap enough to be contained within the bounding parent sphere. The volume of these fragments equal that of the parent sphere, hence ensuring conservation of mass. The axis joining the new spheres is aligned in the direction of the minor principal stress for the parent sphere. The simulation procedure initially involves applying a small stress increment, after this all particles are checked and allowed to break if the particle octahedral shear stress exceeds the particle strength. Following the replacement of the 'breaking' particles, a number of computational 
timesteps are then completed to allow the overlapping fragments to move apart. These two steps of breaking and dissipation are repeated if necessary, until no further breakages occur, after which the stress is reapplied (if necessary), then the next stress increment is applied.

\section{Results}

\subsection{Normal Compression}

In their previous work, the authors modelled normal compression using a scaled-down oedometer. In this work, a sample with a different aspect ratio is used to accommodate the triaxial shearing that follows unloading (shown in Figure 1). The initial sample is mono-disperse, and the initial $d_{0}=2 \mathrm{~mm}$ particles have a characteristic strength of $q_{0}=37.5 \mathrm{MPa}$. Full details of the new sample used in these simulations are provided in Table 1. The one-dimensional and isotropic normal compression lines are given in Figure 2. The slopes for both compression lines are approximately 0.5 , consistent with previous work, and in agreement with both experimental data [14] and Equations (1) and (4) using $m=3.3$.

The progressive particle size distribution (PSD) of the isotropically compressed sample is shown in Figure 3(a). The PSD develops as the stress increases and crushing continues, leading to continuously smaller fragments and an increasing range of particle sizes. McDowell and de Bono [1] provided an in-depth analysis of the evolving fractal particle size distribution during one-dimensional compression, including analysis of the particle contacts. They demonstrated the fractal nature of the evolving PSD by plotting the quantity of each 'sieve' size versus particle size on log-log axes (in which fractal PSDs appear linear). A similar plot for the isotropic simulation is given in Figure 3(b), in which the emergence of a fractal particle size distribution can be observed. At $20 \mathrm{MPa}$, the rightmost two data points give a fractal dimension of 2.3 (obtained from the gradient), while at $28 \mathrm{MPa}$ (the terminal applied stress), the three rightmost data points reveal a fractal dimension of 2.5. 
Figure 4 shows the evolution of the lateral earth pressure coefficient, $K_{0}\left(=\sigma_{\mathrm{h}} / \sigma_{\mathrm{v}}\right)$ for the onedimensional compression test, and a value of approximately 0.68 appears to develop after fractal crushing. This value is higher than typical values for real sand, due to the use of spheres in the simulations, which are not very good at supporting their own weight due to lack of interlock (see [6] for simulations with non-spherical particles). The purpose here is simply to establish whether a constant value of $K_{0}$ emerges during normal compression, and how it relates to the 'strength' of the material. $K_{0}$ is dimensionless - so for self-similar, fractal distributions of particles, it would seem reasonable for $K_{0}$ to be constant. However, the question arises as to what soil parameters $K_{0}$ should be a function of-which must also be dimensionless. An obvious parameter is the critical state friction angle, which we are assuming to be a material constant.

The well-known Jâky [18] equation relates $K_{0}$ to an angle of shearing resistance:

$$
K_{0}=1-\sin \phi
$$

Wood [19] presents an interesting discussion regarding the angle in this equation. For normally consolidated clay it is widely accepted that the angle of friction in (5) should be $\phi_{\text {crit }}$ [19]. However, for sand, Wood [19] suggests that $K_{0}$ depends on the in-situ structure of the sand and so should be expected to correlate with the peak angle of shearing resistance, i.e. $\phi_{\max }$ which is a function of the initial relative density and structure (influenced by particle shape) of the sand at low stress levels. Adding to this conjecture, if one considers a dry stone wall, in which angular pieces of stone are placed together forming a vertical wall, the angle of repose is $90^{\circ}$, giving $K_{0}=0$. The wall supports its own weight perfectly with no horizontal stress because of the extreme particle interlock. However, beyond yield on the normal compression line, as particles crush and a fractal PSD emerges, $\phi_{\max }$ would not be the appropriate parameter to use in Equation (5). It is well known that both clay and sand form very similar normal compression lines beyond yield, albeit at different stress levels. It seems unlikely that these would arise from completely different processes. We therefore propose 
that for normally compressed sand, $\phi_{\text {crit }}$ is the correct parameter to use in Jâky's equation, as is the case for normally consolidated clays (note that for a clay sedimenting out from a slurry before yield and normal compression, as for the sand at low stress levels, $\phi_{\text {crit }}$ would not be appropriate in this case). To test the hypothesis that $\phi_{\text {crit }}$ is the appropriate angle of friction in (5) for a normally compressed sand, using the value of $K_{0}=0.68$ obtained above gives $\phi_{\text {crit }}=19^{\circ}-$ which can be used to predict the critical state dissipation constant $M$, using [20]:

$$
M=\frac{6 \sin \phi}{3-\sin \phi}
$$

which yields a value of $M=0.73$. This will be compared to the value of $M$ (the ratio of deviatoric stress to mean stress at a critical state) obtained from triaxial shear test simulations in the following section, to ascertain whether $K_{0}=1-\sin \phi_{\text {crit }}$ for normally compressed sand well beyond yield.

\subsection{Triaxial Shearing}

Figure 5 shows the normal compression line for the isotropic simulation together with an unloading curve from an isotropic stress of $20 \mathrm{MPa}$. If the particles could not break, there would be no clear transition from elastic to plastic behaviour, and no distinct unloading line would be observed, as is the case for example in Minh and Cheng [12]. The sample at $20 \mathrm{MPa}$ was unloaded to stresses of $10 \mathrm{MPa}$, $8 \mathrm{MPa}, 6 \mathrm{MPa}, 4 \mathrm{MPa}, 2 \mathrm{MPa}$ and $1 \mathrm{MPa}$ before conducting conventional triaxial shear tests. During shearing, the confining pressure was kept constant and strain-control was used to apply the deviatoric stress, such that, the top plate was moved downwards at a constant velocity until the axial strain increment reached $0.01 \%$. The velocity of all the boundaries were then set to 0 , at which point the particles were allowed to break, i.e. the octahedral shear stresses and randomly distributed strengths of all particles were checked, and any particle in which the stress exceeded its strength was replaced by two new fragments. Similar to the normal compression simulations, this was followed by a number of computational timesteps to allow the overlapping fragments to move 
apart, and the induced elastic energy to dissipate; this was repeated until no further fragments were produced, and then the next strain increment was applied.

Figure 6 shows the stress-strain plots for the conventional (constant confining pressure) triaxial tests. The first thing to note is that the ultimate stress ratio in all simulations is approximately $0.75-$ 0.80 , in agreement with the predicted value of 0.73 above using Equations (5) and (6). For the test sheared at $10 \mathrm{MPa}$, there is significant scatter; this is due to both the significant breakage occurring and the relatively small sample. As discussed above, the results here suggest that for a soil which has been normally compressed well beyond yield, the angle in Equation (5) is indeed the critical state angle $\phi_{\text {crit. }}$ To the authors' knowledge, this is a new finding which has shed important light on the application of the Jâky equation (5) to sand. The results also demonstrate consistency between the one-dimensional, isotropic, and triaxial simulations. The conventionally sheared sample at $1 \mathrm{MPa}$ exhibits a peak strength and dilation to a critical state. In fact for all samples up to and including an initial stress of $6 \mathrm{MPa}$, the peak stress ratio and dilation show little variation. Beyond $6 \mathrm{MPa}$, as the isotropic stress increases the peak strength (stress ratio) and dilation reduce. The volumetric strain of the sample subjected to shearing at $8 \mathrm{MPa}$ confining pressure fluctuated around 0 , with no peak stress ratio observed. So although this sample was 'drained' it showed little volume change and therefore must be close to a critical state. The sample subjected to conventional shearing at $10 \mathrm{MPa}$ contracts to a critical state and exhibits no peak strength.

An additional set of simulations were performed after unloading to 2, 4, 6, 8 and $10 \mathrm{MPa}$, this time maintaining the mean effective stress $p^{\prime}$ as constant. This was done using the servo control for the required confining stress and strain control for the application of the deviatoric stress. These tests were performed to achieve additional critical states and provide insight into the behaviour under different stress paths. Figure 7 shows the corresponding plots for the constant- $p^{\prime}$ tests. All of the simulations in this case demonstrate dilation and peak strengths, and reveal the same ultimate 
stress ratio of approximately 0.75 . The dilation to critical states and is further examined below and compared to the behaviour under conventional shearing.

\subsection{Critical State Line}

Figure 8 shows the locations of the final critical states from all triaxial simulations, with arrows indicating the stress paths followed. The critical state line can be seen to be curved in $\log e-\log p$ space, and appears to become approximately parallel to the isotropic normal compression line at high stresses, in agreement with the conventional Critical State Soil Mechanics framework [21]. The observation that at low stresses, the CSL is not parallel with the NCL has been observed experimentally [e.g. 19]. The parallel nature of the emerging critical state line and the established normal compression line not only agrees with the notion that the CSL is parallel (at high stresses) with the NCL [17], but also agrees with the compression law proposed by McDowell and de Bono [1], which predicts a slope of 0.5 for this sand.

It is reassuring to see that the critical states obtained from both sets of shear tests lie on the same critical state line. Moreover, it is interesting to observe the difference in behaviour of the two different shear tests conducted from initial states of $p^{\prime}=8$ and $10 \mathrm{MPa}$. The sample subjected to conventional shearing from $8 \mathrm{MPa}$, which exhibited approximately $0 \%$ volumetric strain, was indeed close to critical. The sample subjected to constant- $p^{\prime}$ shearing from $8 \mathrm{MPa}$ however, dilated to a critical state, as can be seen in Figure 8. An even starker contrast can be observed between the two samples sheared from $p^{\prime}=10 \mathrm{MPa}$, in which the conventionally sheared sample (in which the mean stress increases), undergoes significant contraction to reach the CSL, whereas the constant- $p^{\prime}$ sample dilates to reach a critical state.

From these results, particularly the conventional triaxial tests, which exhibited ductile behaviour and contraction at the highest stresses, it can be therefore seen that fractal distribution of particles which resulted from crushing during normal compression (in this case to $20 \mathrm{MPa}$ ) is the key to 
explaining the behaviour of overconsolidated sands on the dense and loose sides of critical.

However, although Figure 8 suggests a unique critical state line, if the same sample was unloaded, 'reconstituted' to a looser state, then re-compressed and again unloaded to various overconsolidation ratios, one would expect a different critical state line to be observed upon shearing, particularly at low stresses [e.g. 22,23 ]. That is to say the critical state line observed in Figure 8 is not fixed, but rather is a function of the crushing that has occurred, i.e. the particle size distribution achieved from isotropic compression to $20 \mathrm{MPa}$. The triaxial shear tests presented above all start from an identical particle size distribution (shown in Figure 3). It is also important to note that although Coop et al. [24] showed that crushing and volume change can occur in ring shear tests to shear strains over the order of $1000 \%$, what we are examining here is the conventional notion of a critical state [18] at around $15-20 \%$ strain at which the volume and effective stresses are approximately constant.

\section{Conclusions}

A previously used particle splitting model has been used to investigate the evolution of critical states. A sample of spheres has been isotropically compressed to a stress of $20 \mathrm{MPa}$, well beyond yield and such that a fractal distribution of particles has evolved. The sample has been isotropically unloaded to various stress levels and then conventional and constant- $p^{\prime}$ triaxial shear tests have been performed. All samples reach a critical state with a critical state constant which is consistent with the predicted value using the measured value of $K_{0}$ under one-dimensional normal compression, suggesting that for sand that is normally compressed beyond yield, $K_{0}=1-\sin \phi_{\text {crit. }}$ At low stress levels the samples exhibit a peak strength and dilation, which both reduce as the isotropic stress increases. The conventional triaxial test from $8 \mathrm{MPa}$ exhibits little volume change even though drained and thus has been established to be close to critical, while that sheared from $10 \mathrm{MPa}$ exhibits ductile behaviour and contraction to a critical state. The constant- $p^{\prime}$ tests shearing from these same states however both dilated in order to reach the critical state line. The particle splitting 
model has therefore been able to capture the essential behaviour of overconsolidated sands and a critical state line has been established, which appears to be parallel to the isotropic normal compression line at high stresses in agreement with the conventional Critical State Soil Mechanics framework. Further work will continue examining the behaviour of the overconsolidated samples under different stress paths, obtaining further points on the CSL at high stress levels and using larger samples (a computationally very demanding task), and focus on establishing the critical state line for samples unloaded from different isotropic stresses. In addition simulations will be performed on 'reconstituted normally compressed sands' to examine the on-uniqueness of the critical state line, as well as further work incorporating particle shape and therefore obtaining more realistic values of $K_{0}$.

\section{Acknowledgements}

The authors would like to thank the Engineering and Physical Sciences Research Council (EPSRC) for their financial support. 


\section{Notation}

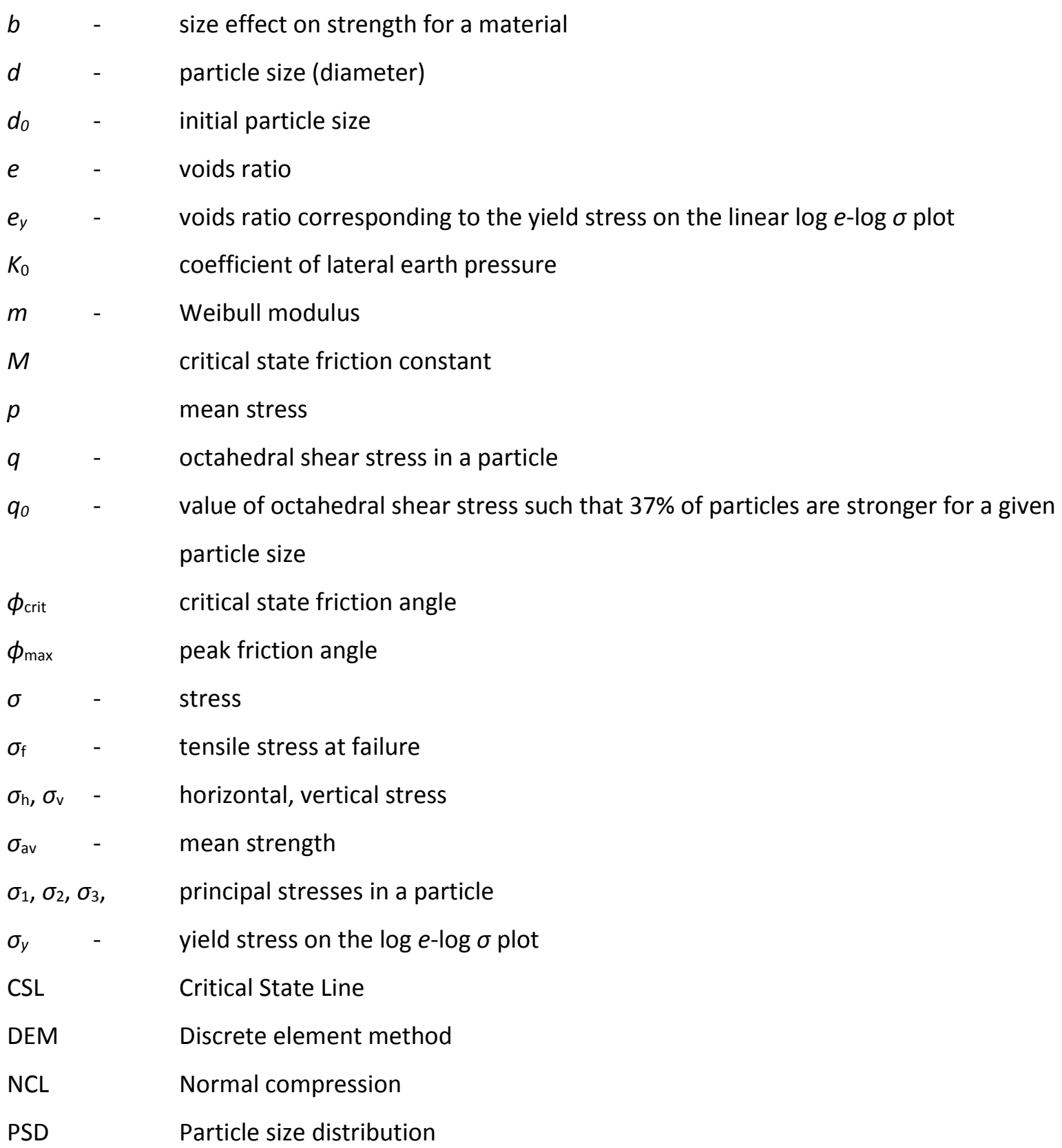




\section{References}

[1] G.R. McDowell, J.P. de Bono, On the micro mechanics of one-dimensional normal compression, Géotechnique. 63 (2013) 895-908. doi:10.1680/geot.12.P.041.

[2] G.R. McDowell, J.P. de Bono, P. Yue, H.-S. Yu, Micro mechanics of isotropic normal compression, Géotechnique Lett. 3 (2013) 166-172. doi:10.1680/geolett.13.00050.

[3] J.M. Pestana, a. J. Whittle, Compression model for cohesionless soils, Géotechnique. 45 (1995) 611-631. doi:10.1680/geot.1995.45.4.611.

[4] G.R. McDowell, A physical justification for log e-log $\sigma$ based on fractal crushing and particle kinematics, Géotechnique. 55 (2005) 697-698. doi:10.1680/geot.2005.55.9.697.

[5] J.P. de Bono, G.R. McDowell, DEM of triaxial tests on crushable sand, Granul. Matter. 16 (2014) 551-562. doi:10.1007/s10035-014-0500-x.

[6] J.P. de Bono, G.R. McDowell, An insight into the yielding and normal compression of sand with irregularly-shaped particles using DEM, Powder Technol. 271 (2015) 270-277. doi:10.1016/j.powtec.2014.11.013.

[7] J. a. Åström, H.J. Herrmann, Fragmentation of grains in a two-dimensional packing, Eur. Phys. J. B. 5 (1998) 551-554. doi:10.1007/s100510050476.

[8] S. Lobo-Guerrero, L.E. Vallejo, Crushing a weak granular material: experimental numerical analyses, Géotechnique. 55 (2005) 245-249. doi:10.1680/geot.2005.55.3.245.

[9] O. Harireche, G.R. Mcdowell, Discrete element modelling of yielding and normal compression of sand, Géotechnique. 52 (2002) 299-304. doi:10.1680/geot.2002.52.4.299.

[10] Y.P. Cheng, M.D. Bolton, Y. Nakata, Discrete element simulation of crushable soil, Géotechnique. 53 (2003) 633-641. doi:10.1680/geot.2003.53.7.633.

[11] Y.P. Cheng, M.D. Bolton, Y. Nakata, Grain crushing and critical states observed in DEM simulations, in: Powders Grains 2005, 2005: pp. 1393-1397.

http://discovery.ucl.ac.uk/11099/.

[12] N.H. Minh, Y.P. Cheng, A DEM investigation of the effect of particle-size distribution on onedimensional compression, Géotechnique. (2013) 44-53. doi:10.1680/geot.10.P.058.

[13] M.D. Bolton, Y. Nakata, Y.P. Cheng, Micro- and macro-mechanical behaviour of DEM crushable materials, Géotechnique. 58 (2008) 471-480. doi:10.1680/geot.2008.58.6.471.

[14] G.R. McDowell, On the yielding and plastic compression of sand, Soils Found. 42 (2002) 139145.

[15] J.C. Jaeger, Failure of rocks under tensile conditions, Int. J. Rock Mech. Min. Sci. Geomech. Abstr. 4 (1967) 219-227. doi:10.1016/0148-9062(67)90046-0. 
[16] Itasca, Particle Flow Code in 3 Dimensions, Itasca Consulting Group, Inc., Minneapolis, Minnesota, 2005.

[17] G.R. McDowell, A. Amon, The application of weibull statistics to the fracture of soil particles, Soils Found. 40 (2000) 133-141.

[18] J. Jaky, Pressure in silos, in: Proc. 2nd Int. Conf. Soil Mech. Found. Eng. Vol 1., 1948: pp. 103107.

[19] D.M. Wood, Soil Behaviour and Critical State Soil Mechanics, Cambridge University Press, 1990.

[20] M.D. Bolton, A Guide to Soil Mechanics, Macmillan Press Ltd, London, 1979.

[21] A. Schofield, P. Wroth, Critical State Soil Mechanics, McGraw-Hill, London, 1968.

[22] D.M. Wood, K. Maeda, Changing grading of soil: Effect on critical states, Acta Geotech. 3 (2008) 3-14. doi:10.1007/s11440-007-0041-0.

[23] V. Bandini, M.R. Coop, The influence of particle breakage on the location of the critical state line of sands, Soils Found. 51 (2011) 591-600.

[24] M.R. Coop, K.K. Sorensen, T. Bodas Freitas, G. Georgoutsos, Particle breakage during shearing of a carbonate sand, Géotechnique. 54 (2004) 157-163. doi:10.1680/geot.2004.54.3.157. 
Table 1. Input parameters for compression and triaxial simulations.

\begin{tabular}{|l|l|}
\hline Input Parameter & Value \\
\hline Initial Particle Diameter, $d_{0}(\mathrm{~mm}):$ & 2 \\
\hline Particle Density: $\left(\mathrm{kg} / \mathrm{m}^{3}\right)$ & 2650 \\
\hline Initial Voids Ratio, $e_{0}:$ & 0.82 \\
\hline Initial No. of Particles: & 390 \\
\hline Shear Modulus, $G(\mathrm{GPa}):$ & 28 \\
\hline Poisson's Ratio, $v$ & 0.25 \\
\hline Particle Friction Coefficient & 0.5 \\
\hline Wall Friction Coefficient & 0.0 \\
\hline Weibull Modulus, $m:$ & 3.3 \\
\hline $37 \%$ Strength, $q_{0}\left(\right.$ for $\left.d_{0}\right)(\mathrm{MPa}):$ & 37.5 \\
\hline Sample Height $(\mathrm{mm}):$ & 18 \\
\hline Sample Diameter $(\mathrm{mm}):$ & 15 \\
\hline
\end{tabular}

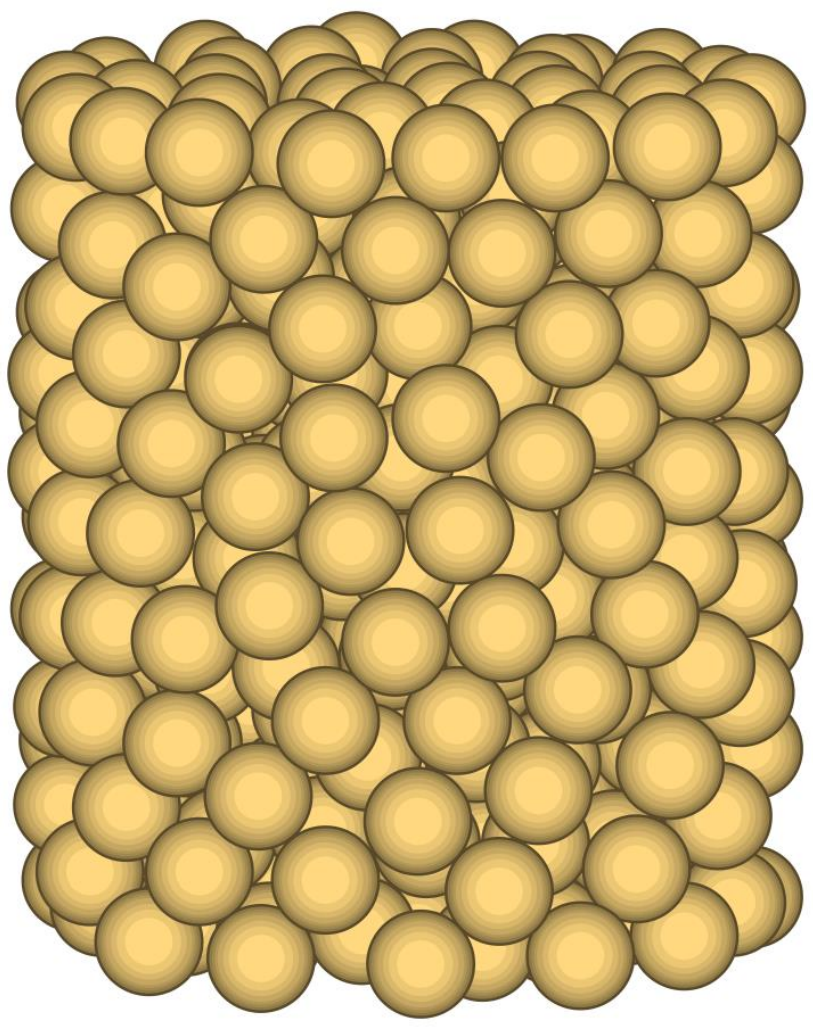

Figure 1. Initial sample used for compression and shearing. 


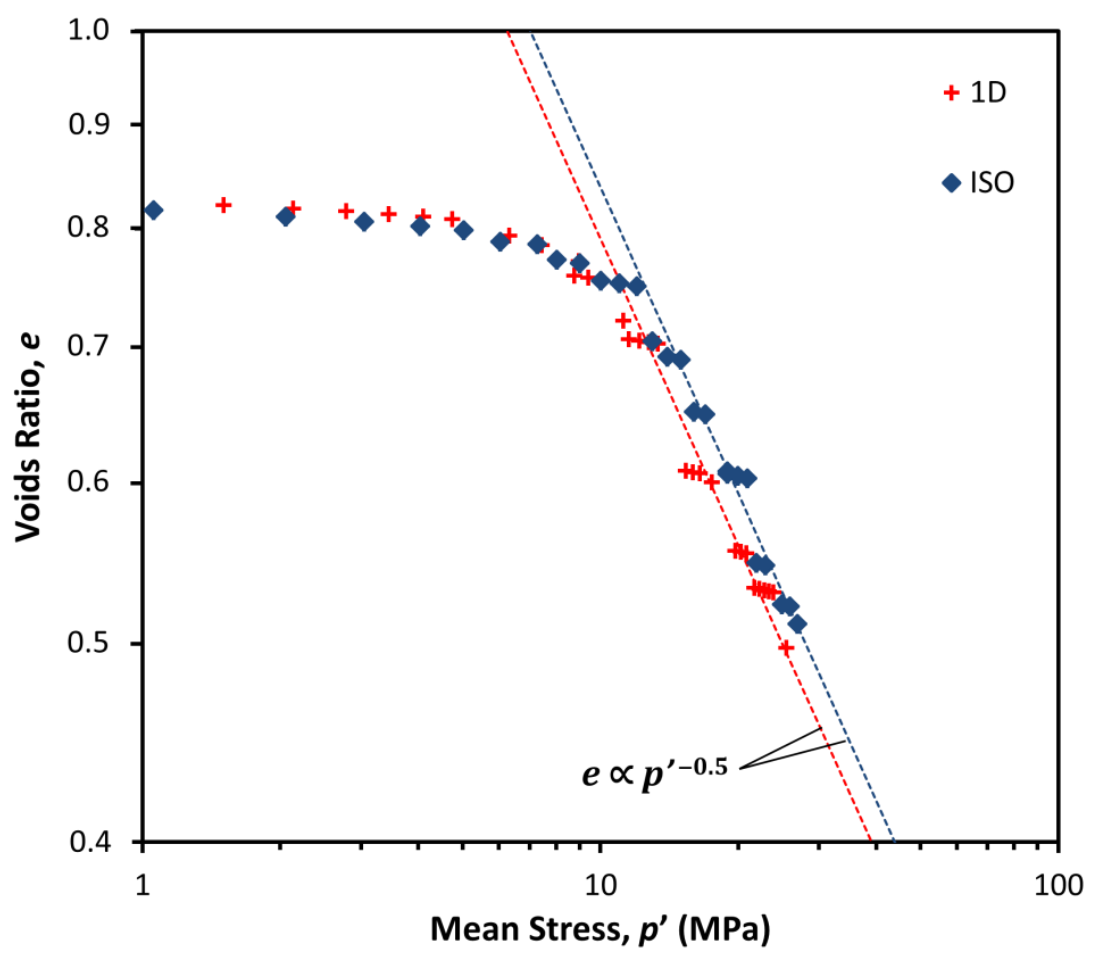

Figure 2. One-dimensional and isotropic compression behaviour. 


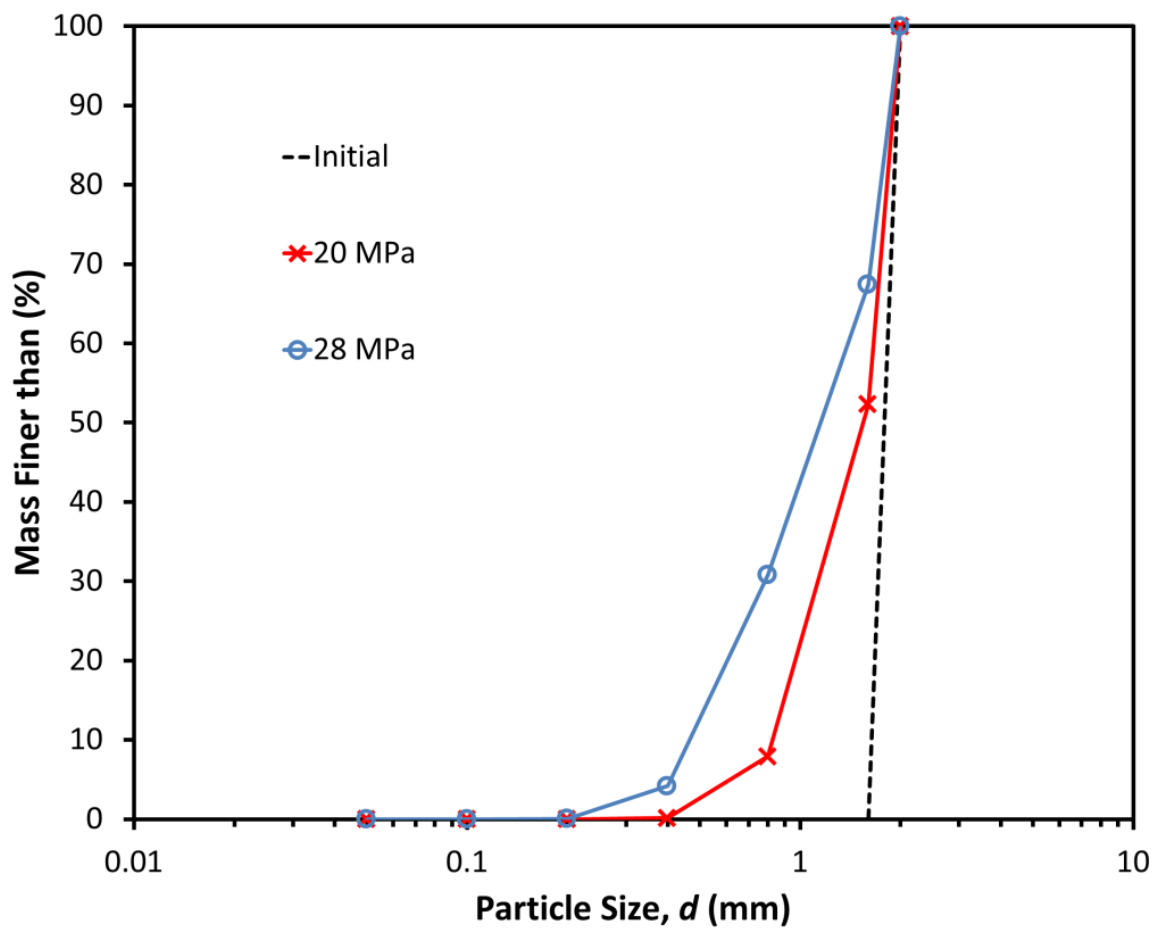

(a)

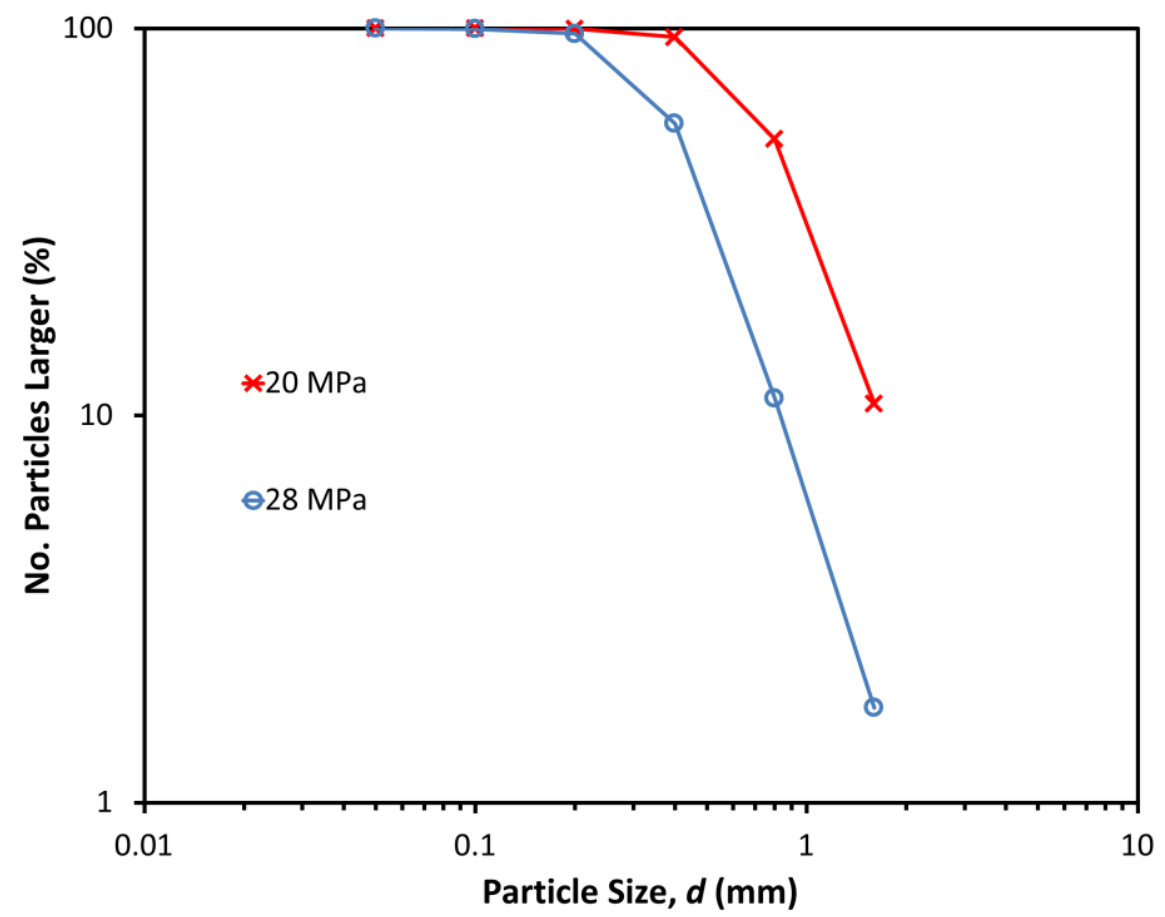

(b)

Figure 3. Evolution of particle size distribution during isotropic compression. Conventional plot (a) and number of particles versus size (b). 


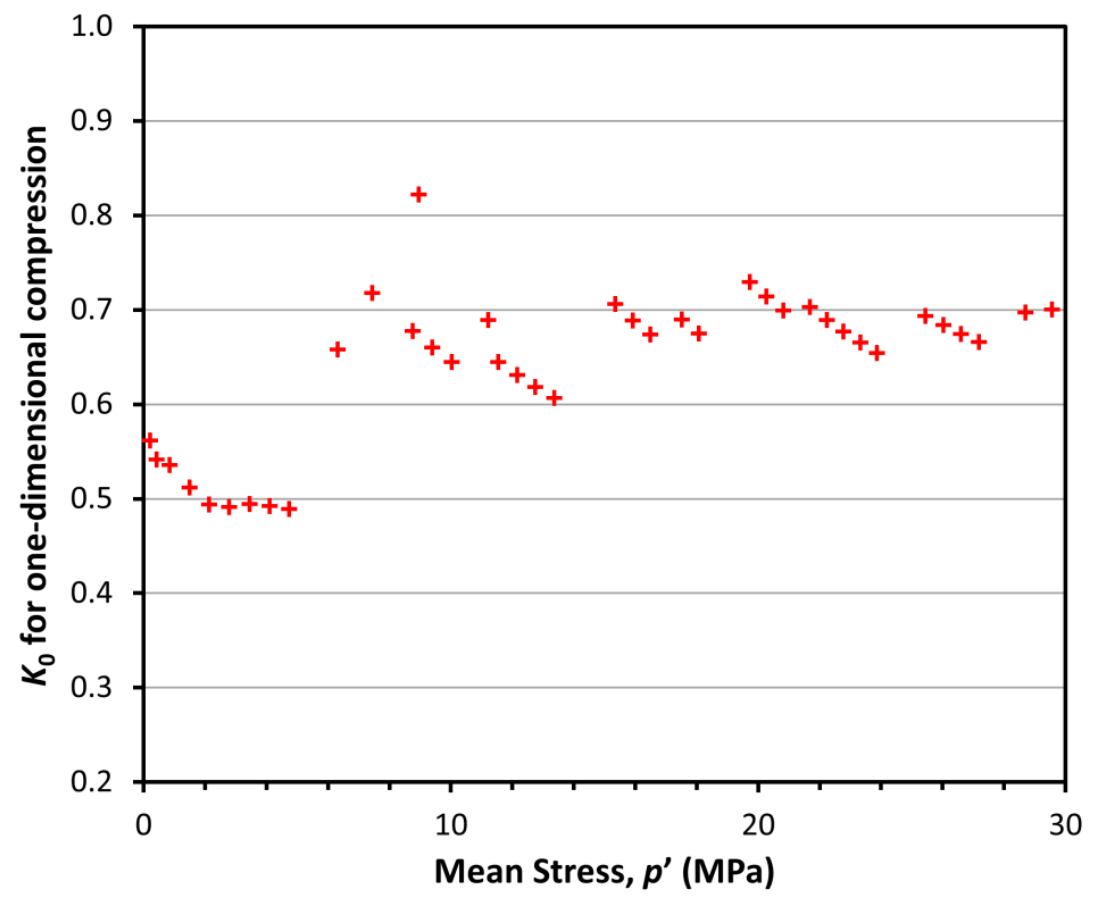

Figure 4. Evolution of the coefficient of lateral earth pressure during one-dimensional compression.

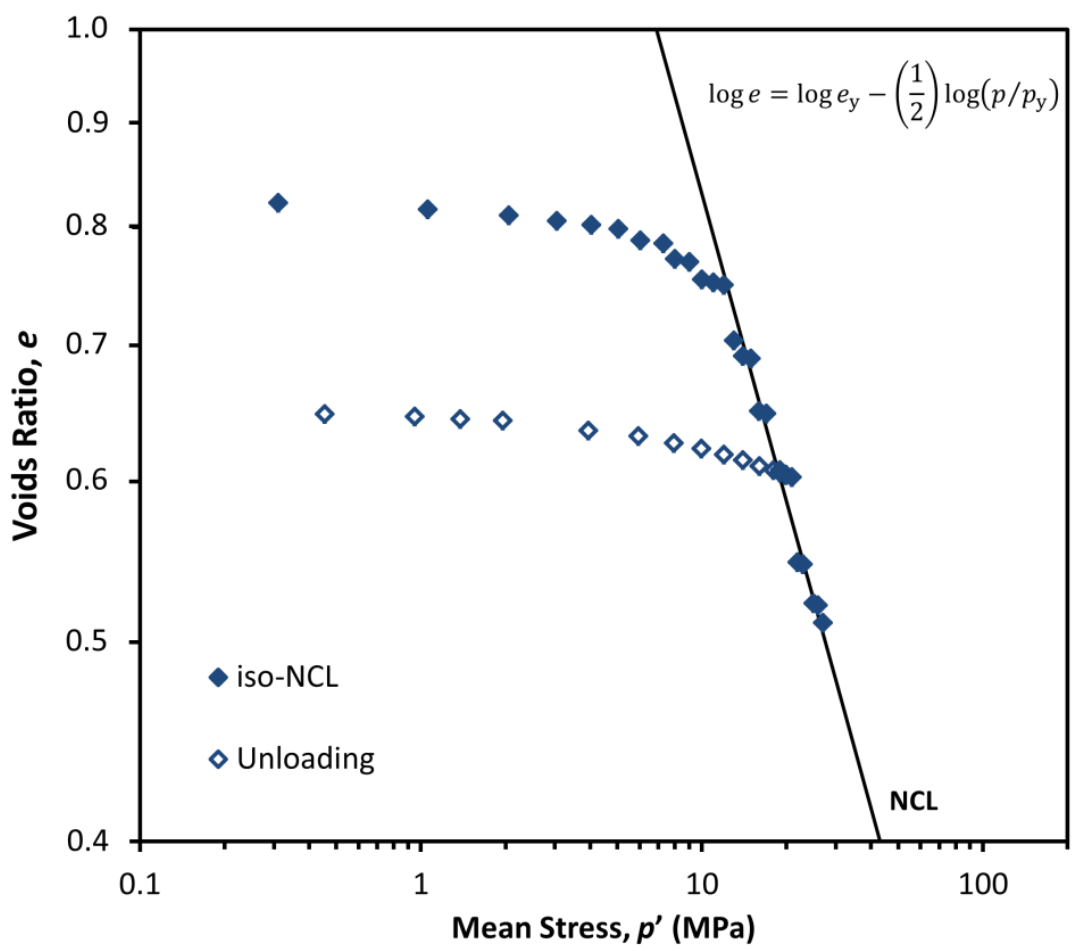

Figure 5. Isotropic compression and unloading behaviour. 


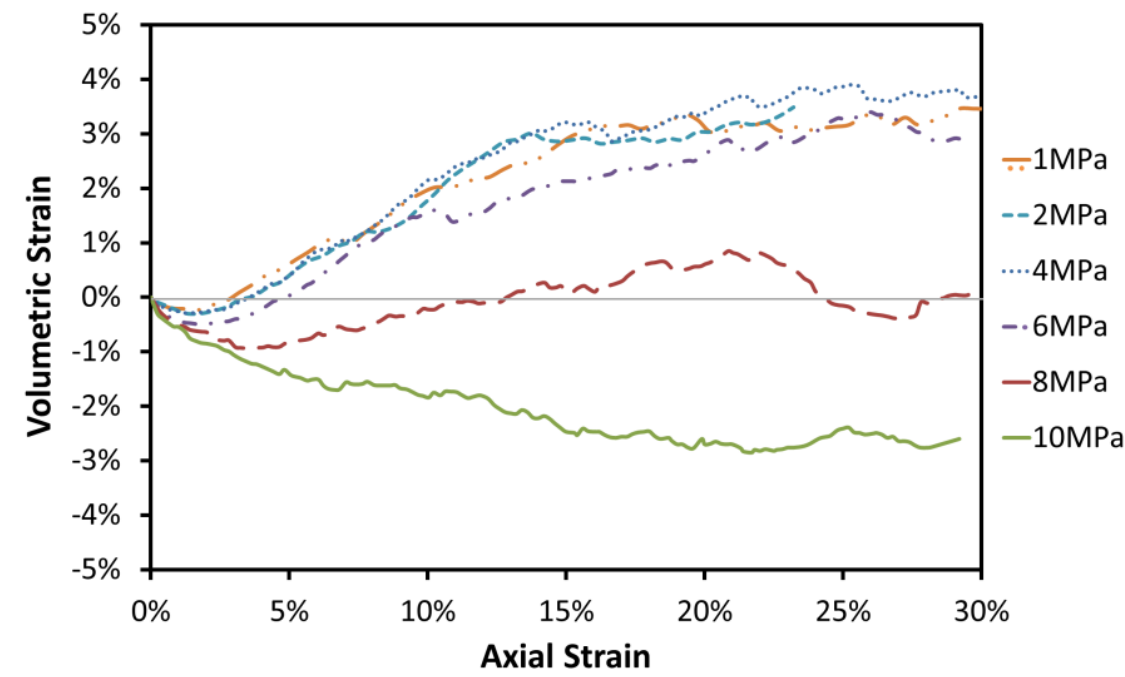

(a)

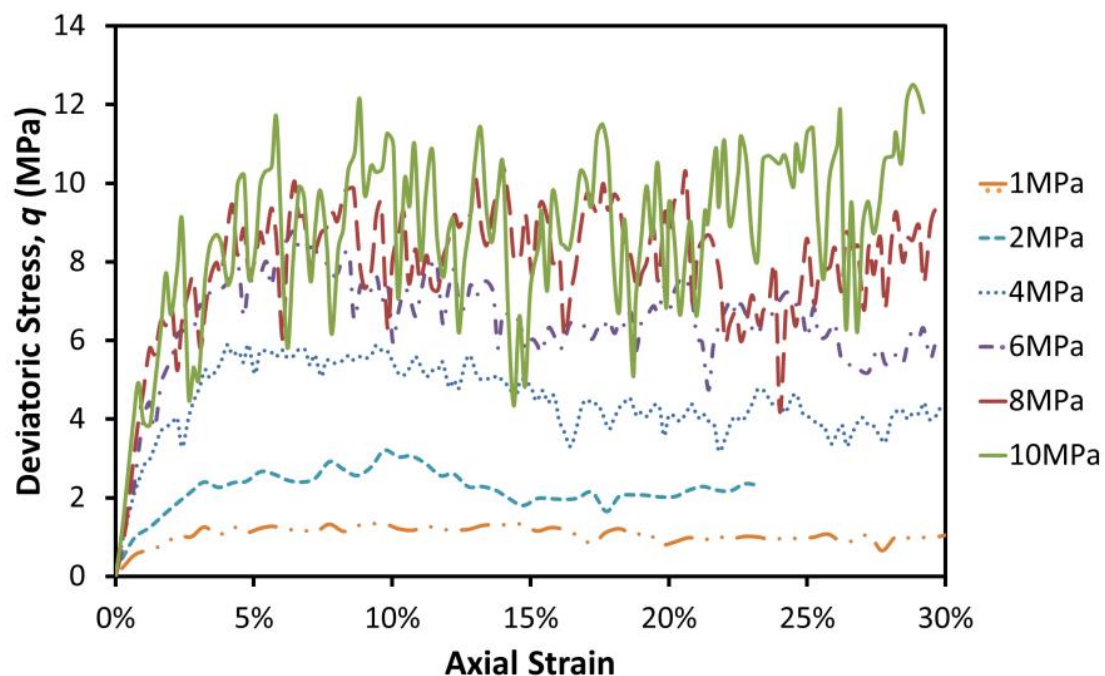

(b)

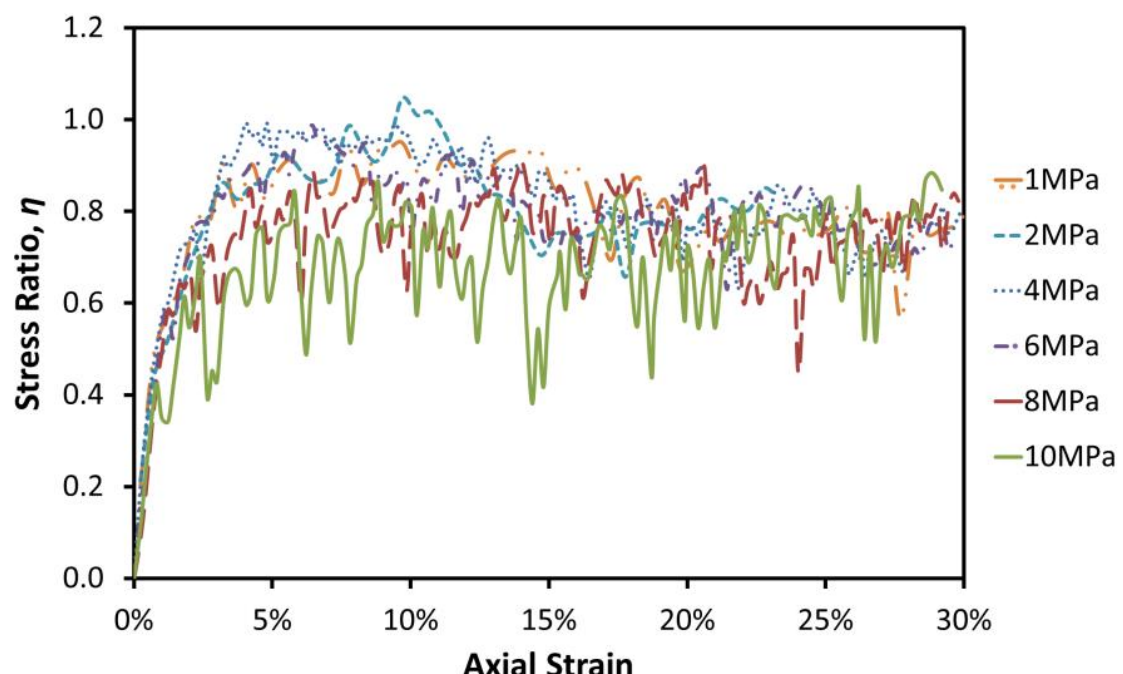

(c)

Figure 6. Stress-strain behaviour for conventional triaxial tests performed after unloading to a range of isotropic stresses. 


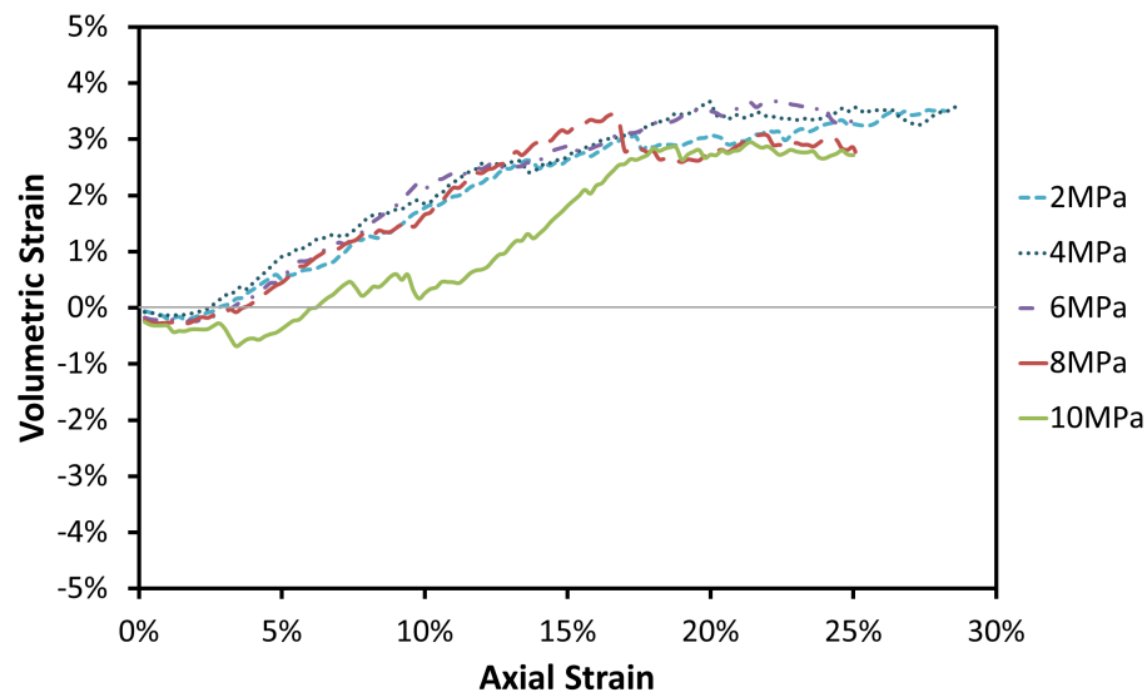

(a)

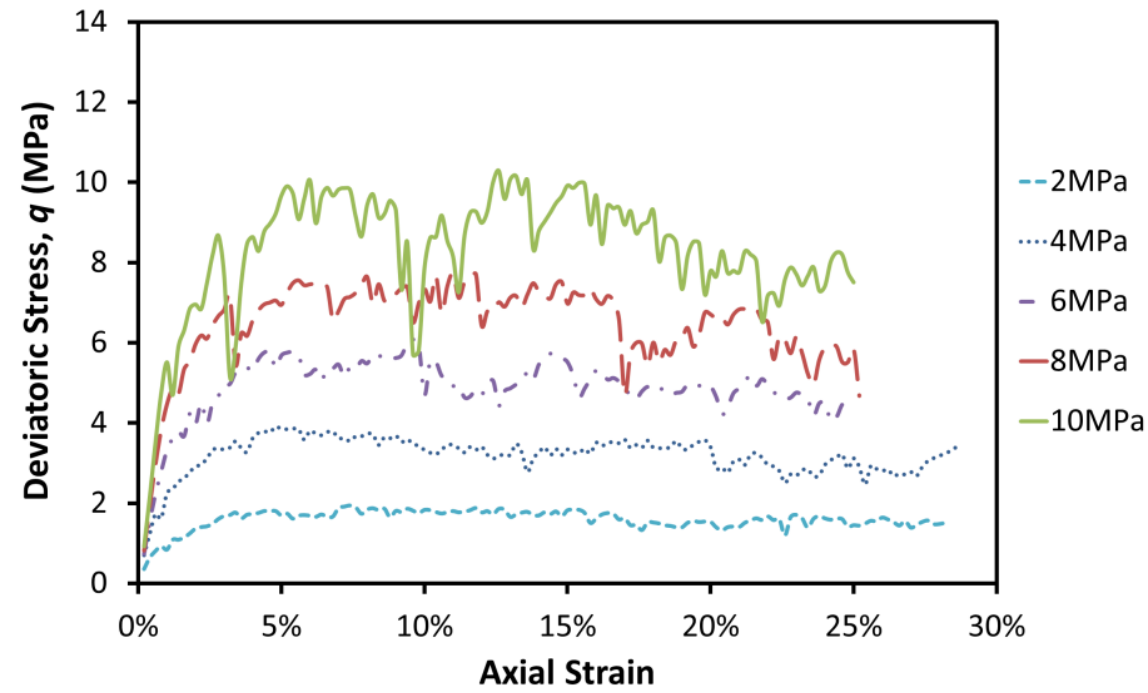

(b)

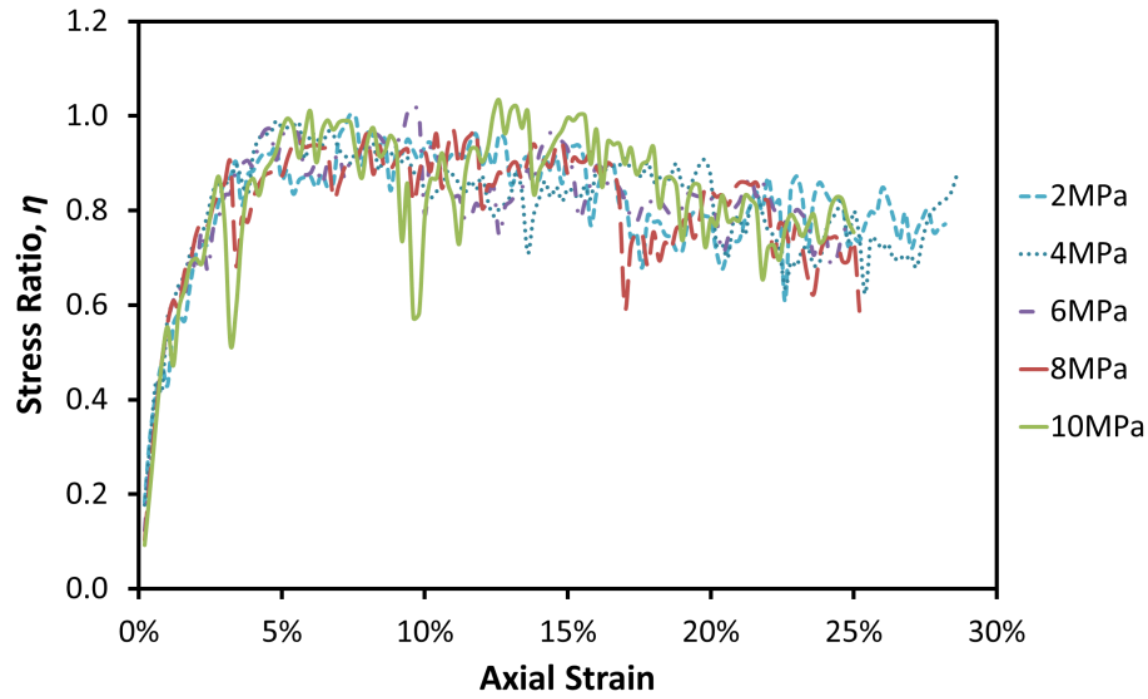

(c)

Figure 7. Stress-strain behaviour for constant-p' triaxial tests performed after unloading to a range of isotropic stresses. 


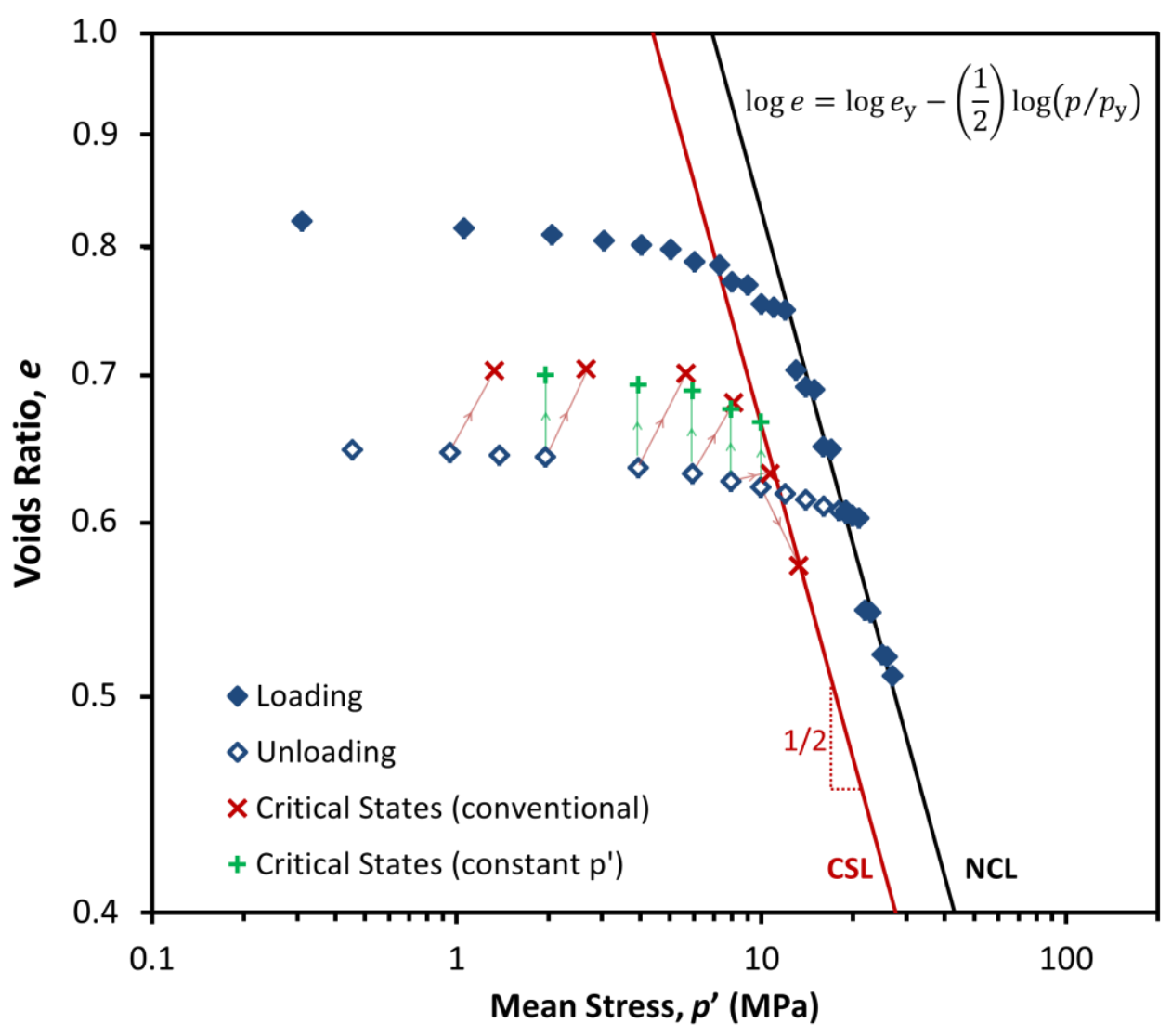

Figure 8. Critical states. 